\title{
Flow field investigation behind a trapezoidal rib and the effect of the synthetic jet
}

\author{
SUSHANTA DUTTA* and ABHISHEK MALIK \\ Mechanical and Industrial Engineering Department, Indian Institute of Technology Roorkee, Roorkee, India \\ e-mail: duttafme@iitr.ac.in
}

MS received 18 May 2019; revised 6 September 2019; accepted 9 September 2019

\begin{abstract}
The motive of the present work is to investigate the combined effect of synthetic jet and chamfer angle $\left(15^{\circ}, 18^{\circ}\right.$ and $\left.21^{\circ}\right)$ of a trapezoidal rib on flow phenomena. However, detailed study is restricted to $21^{\circ}$ chamfer angle, which is the optimum angle from the heat transfer perspective. The rib has an obstruction of 5\% of the tunnel for an aspect ratio of 30. Emphasis has been given to identifying the optimal location of the synthetic jet at which the recirculation zone behind the rib turbulator becomes a minimum. Experiments are performed on a low-velocity subsonic wind tunnel by particle image velocimetry (PIV) and hotwire anemometry (HWA). Flow field has been characterized in terms of time-averaged velocity magnitude, vorticity, streamlines, turbulence quantities such as turbulence intensity, Reynolds stress and root mean square (rms) velocities as well as instantaneous flow field. The data are presented at Reynolds number 32,000, based on the hydraulic diameter of the test section. Along with the large primary recirculation, a secondary recirculation bubble is visible at the leeward corner predominantly for the angles $18^{\circ}$ and $21^{\circ}$. The secondary bubble is considered to be responsible for maximum heat transfer from the near rib surface. The synthetic jet is placed at different upstream and downstream locations to see the effect on flow parameters. With the synthetic jet, the primary recirculation bubble moves closer to the rib, which ultimately reduces the recirculation length. A greater mixing is observed in the near wake of the obstacle, and the secondary recirculation zone near the surface diminishes. Out of six different synthetic jet locations studied, the maximum reduction of the recirculation bubble is observed when the synthetic jet is placed $0.5 D$ upstream and $1.5 D$ downstream from the rib front face. A comparison has been made between with and without synthetic jet for these flow parameters.
\end{abstract}

Keywords. Synthetic jet; PIV; HWA; trapezoidal rib; recirculation length.

\section{Introduction}

Flow control using a synthetic jet (SJ) is a very new technique and emerges as a promising method for heat transfer enhancement, drag reduction, mixing, etc. An SJ is formed entirely from the fluid in which it is deployed and hence net mass flux is zero. An SJ is a train of vortices formed by ejecting the fluid through a small opening. It has an orifice at one side of the cavity and a diaphragm on another side. Oscillation of diaphragm controls the suction and ejection of the fluid. It has application in cooling integrated circuits as well as micro- and macro-flow control. Due to its compact design, it is very suitable for micro-applications.

Glezer et al [1] were the first to develop the SJ and patent their work in US Patent 1998. They specify it as a train of vortices and give its applications in the field to manipulate the direction of fluid flow. Later, various researchers [2-5] have performed their research on the variation of operating characteristics such as oscillation frequency of diaphragm,

*For correspondence

Published online: 25 October 2019 orifice shape, cavity height, etc. for various applications. Chaudhari et al [2] performed experiments at different operating frequencies, depths of the cavity and orifice diameters to examine the optimum response of the SJ. The resonance frequency of the SJ was found to be the resonance frequency of diaphragm; the result has shown that the input power is a minimum at this frequency and a particular diameter of the orifice gives the maximum velocity. The same first author in a different study performed experiments [6] to apply SJ for enhancement of heat transfer on a flat plate by impingement of SJ on it. Jain et al [7] studied the effect of cavity radius and orifice shape to the SJ operating characteristics numerically. They found that for constant swept volume, the mean stream velocity of the SJ becomes a maximum at optimum cavity radius. However, orifice height affects SJ velocity more rather than cavity depth. Krishnan and Mohseni [8] investigated experimentally and numerically the characteristics of rectangular SJ and compared it to a continuous rectangular jet. Results show that the SJ velocity spreading and the decay rate are increased with the stroke ratio and it is independent of Reynolds 
number. Actuator geometry also affects the operating characteristics of the SJ.

Some researchers applied the SJ for flow separation control and mixing of the fluid. Tang and Zhong [9] used the SJ actuator arrays on the wing model along the chord length at two distinct positions from the leading edge and showed $27.4 \%$ lift coefficient enhancement with $19.6 \%$ drag reduction. Front arrays give more effect than their rear counterpart. Feng and Wang [10] have investigated the modification of wake behind the circular cylinder using the SJ. SJ has been placed at the front stagnation point. The flow field is measured by a time-resolved particle image velocimetry (PIV). The interaction between the SJ and the upcoming flow rises to an envelope form upstream of the circular cylinder, which acts as a virtual aerodynamic shape. Xia and Zhong [11] investigated the effect of SJ on two laminar water streams mixing in a rectangular channel. These authors have used a pair of SJ on the lateral wall surface and kept them $180^{\circ}$ out of phase. Results showed the enhancement of mixing of the fluid due to the SJ.

The rib turbulators have a wide range of applications in various engineering fields. Cooling of gas turbine blades, the internal surface of pipes in a heat exchanger and convective heat transfer in a solar air heater are a few applications. The flow behaviour behind the rib is very complex; with its time-dependent behaviour, it is very challenging to investigate its recirculation zone. This makes this investigation attractive with sophisticated experimental techniques. Tariq et al [12] experimentally investigated the flow behaviour and the heat transfer effect behind the square rib with a slit. They studied five different slit geometries by liquid crystal thermography (LCT) for temperature measurement and smoke visualization. A hotwire anemometer (CTA) and a cold wire anemometer were used to study turbulence and temperature measurement, respectively. Results showed that $20 \%$ slit made the Nusselt number optimum; however, more than $40 \%$ slit behaves like two different ribs. Panigrahi and Acharya [13] experimentally investigated the flow over a rib turbulator in single-frequency and dual-frequency modes. A laser Doppler velocimeter and a hot wire anemometer were used for measurement. Results show that dual frequency permits more shear growth compared with single frequency; however, phase difference does not seem to have much effect on it. The same authors also studied [14] the turbulent behaviour behind a square rib by pattern recognition approach. The author stated that with excitation, the shear layer reattached earlier than before. Ali et al [15] experimentally studied the flow behaviour and heat transfer effect over a trapezoidal rib with different chamfer angles. LCT, PIV and hotwire anemometry (HWA) are used to obtain the results, which state that with an increase in Reynolds number, secondary bubble near the leeward corner diminishes. The authors concluded that reattachment point and maximum heat transfer point do not coincide but differ by $10-12 \%$. It is noticed that the wake behind the $\mathrm{SJ}$ is similar to the wake profile behind surface-mounted obstacles [16]. The bluff body wake was extensively studied by various researchers [17-19], to characterize the wake and related forces. From the literature, it is observed that no data are available on the effect of SJ on the flow field for a surfacemounted obstacle. Also, limited information is available on the flow field over a surface-mounted trapezoidal rib.

The objective of the present study is to investigate the flow behaviour behind the trapezoidal rib with different chamfer angles and examine the effect of SJ on it. As mentioned earlier, the rib turbulators have a wide range of applications in heat transfer enhancement. Similarly, the SJ has been successfully used for drag reduction in the bluff object, mixing and heat transfer. Hence, the present investigation has a broader scope concerning applications.

\section{Experimental set-up}

Figure 1 shows a schematic of the experimental set-up, along with a snapshot of the installation. Experiments are conducted in a horizontal low-speed wind tunnel. A contraction cone (ratio 9:1) along with a settling chamber is used ahead of the test section. The free stream turbulence level $(<0.08 \%)$ and uniform velocity were checked in the tunnel before performing the experiments. The test section is made of a transparent acrylic sheet of cross section $300 \times 200 \mathrm{~mm}^{2}$ and $3.6 \mathrm{~m}$ in length. A diffuser having an angle of divergence $6^{\circ}$ is attached after the test section for pressure recovery. The air flow is drawn in the tunnel using a blower and it is controlled using an $\mathrm{AC}$ frequency inverter (Siemens micromaster 440 drive). Experiments are performed with trapezoidal ribs of three different chamfer angles $15^{\circ}, 18^{\circ}$ and $21^{\circ}$ having aspect ratio 30 and blockage ratio 5\%. The ribs are made of aluminium due to its good machinability and surface finish. They are machined with sharp corners, and black anodizing is performed on their surface to reduce the reflectivity.

The orifice of the SJ (figure 1) is $3 \mathrm{~mm}$ in circular diameter and flashes on the bottom surface of the test section. A speaker of 3-W power and 8-ohm impedance with 3-inch diameter is used to actuate the SJ. Space between the speaker diaphragm and the flat surface of the test section behaves like a cavity of the SJ, which is about $5.7 \mathrm{~cm}^{3}$. The SJ is placed at different locations in the upstream and the downstream of the rib. A function generator (Tektronix AFG 3022B Dual channel) is used to actuate the speaker with a continuous sine wave.

PIV measurements are taken at the centre plane of the SJ along the axis of the test section. A double-pulse Nd:YAG laser (TSI evergreen, $532 \mathrm{~nm}$ ) with maximum energy level of $200 \mathrm{~mJ}$ per pulse is used for PIV measurement. A combination of the spherical and cylindrical lens is used to convert the laser beam into a laser sheet to illuminate the tracer particles. The cylindrical lens controls the height of 


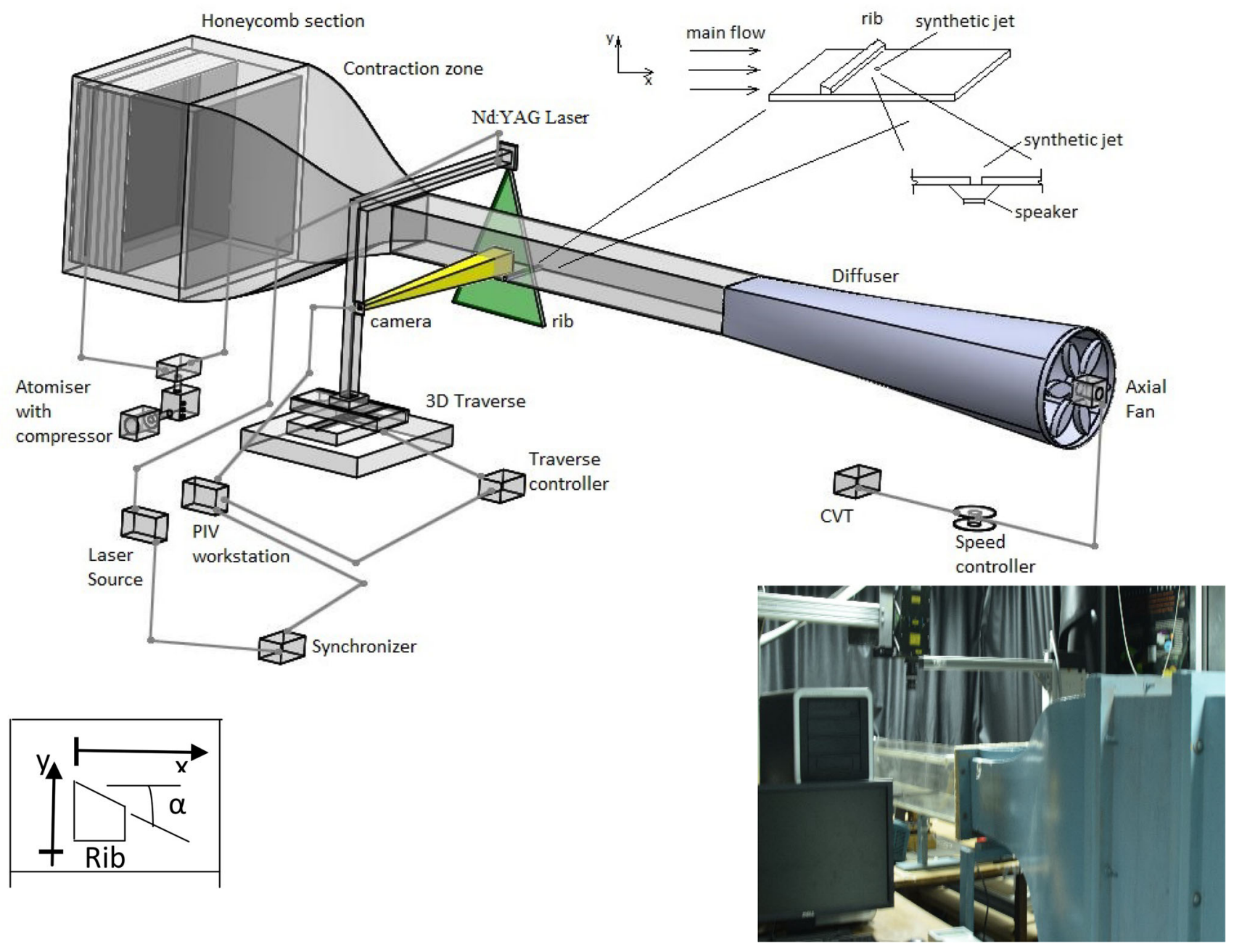

Figure 1. Schematic diagram of experimental set-up, with rib geometry and snapshot image of the experimental set-up.

the sheet while the spherical lens controls the thickness. Minimum thickness of the laser sheet is measured as $0.8 \mathrm{~mm}$ at the waist.

Proper uniform and homogeneous seeding is very important for good quality PIV measurement. In the present study, olive oil is used for seeding purpose due to its nontoxic property and good dispersing quality. An atomizer (TSI Six-Jet Atomizer model 9306) is used to atomize the seeding oil into fine particles (average diameter $2 \mu \mathrm{m}$ ). These tracer particles are mixed with air ahead of the settling chamber by suitable arrangement. The tracer particle images are captured using a 4MP CCD Camera (TSI Power view Plus, model 630159) having frame straddling time of 200 ns with 15 fps frame speed synchronized with the laser via synchronizer (TSI model 610035). A Nikon 70-mm lens is used, which gives $143 \times 143 \mathrm{~mm}^{2}$ field of view. A dualprocessor computer with a frame grabber is used to save and process the PIV images. Depending on the flow velocity, the pulse separation time is set between 100 and $250 \mu \mathrm{s}$. To get smooth time-averaged data, 300 instantaneous image pairs for each set are taken into consideration. A recursive Nyquist grid engine is used due to its excellent accuracy while processing the PIV data.

A hotwire anemometer (Make: Dantec Dynamics) is used to examine the fluctuating components and to validate the PIV data. A 1D probe (55P11) is used for the measurement at three different locations: $4 D, 6 D$ and $11 D$ from the front edge of the rib. Twenty thousand samples are used to obtain the fluctuating component at $1 \mathrm{kHz}$ sample frequency. A bandpass filter $(0.1 \mathrm{~Hz}-1 \mathrm{kHz})$ is used to avoid unwanted noise in the measuring data. The sampling rate was sufficient to capture at least 20 cycles of the dominant frequency.

\section{Uncertainty and data validation}

The present sets of data are validated with published literature to cross-check the authenticity of the data. Figure 2(a) shows the velocity profile behind a square rib at 

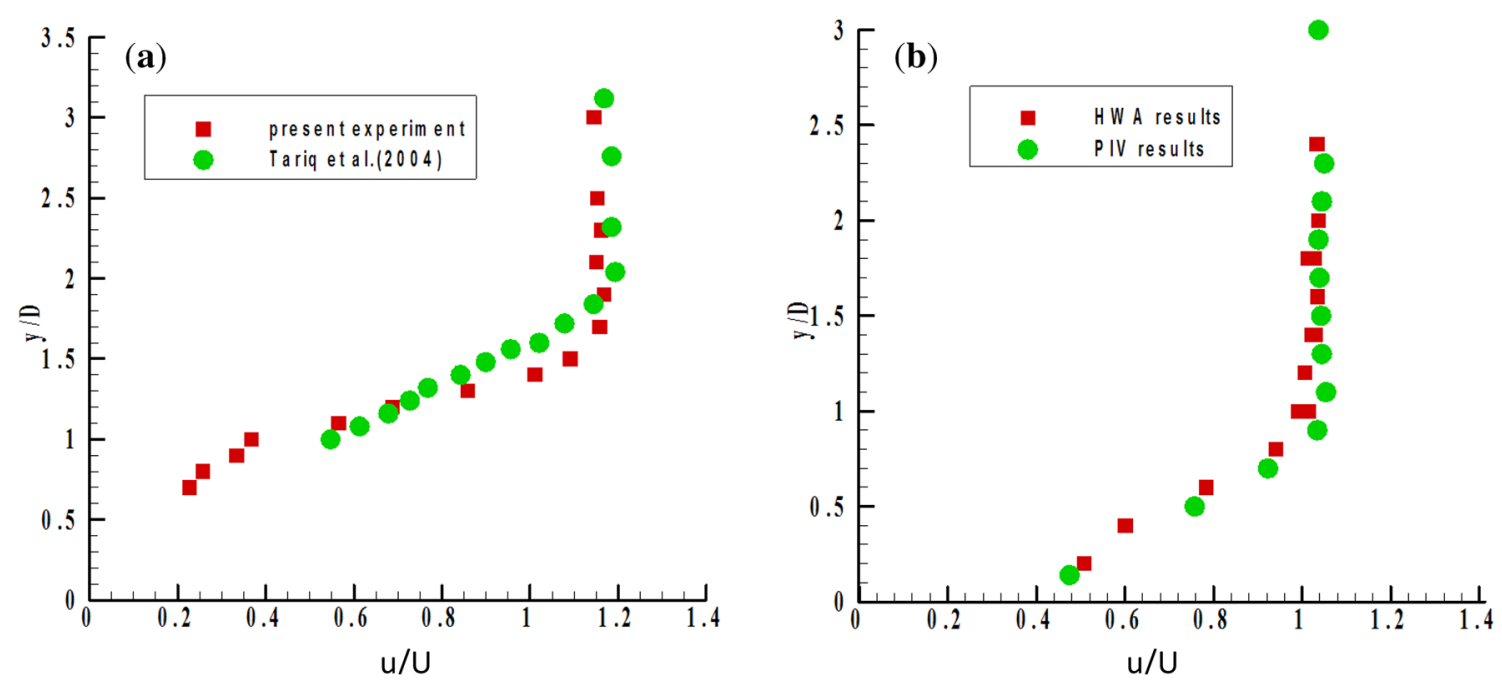

Figure 2. (a) Comparison of velocity profile behind square rib with published data [18]. (b) Comparison of $u$-velocity profile measured in the tunnel by PIV and HWA.

$4 D$ from the front edge of the rib and it compares with Tariq et al [12]. A close match is found between the two results. Figure 2(b) shows the comparison of free stream velocity profile in the tunnel using PIV with HWA for the same plane and results pointed towards the excellent match in between these two techniques.

Figure 3 shows the laminar velocity profile (Blasius equation) for a smooth duct and compared with present PIV data. A good match is found between these two and suggests that a laminar boundary layer prevails in the tunnel for the Reynolds number studied.

The factors responsible for uncertainty in the experimental data are seeding particle size, spurious vectors, density of seeding particles, light illumination, time lag of pulse, etc. The authors quote less than $5 \%$ spurious vector

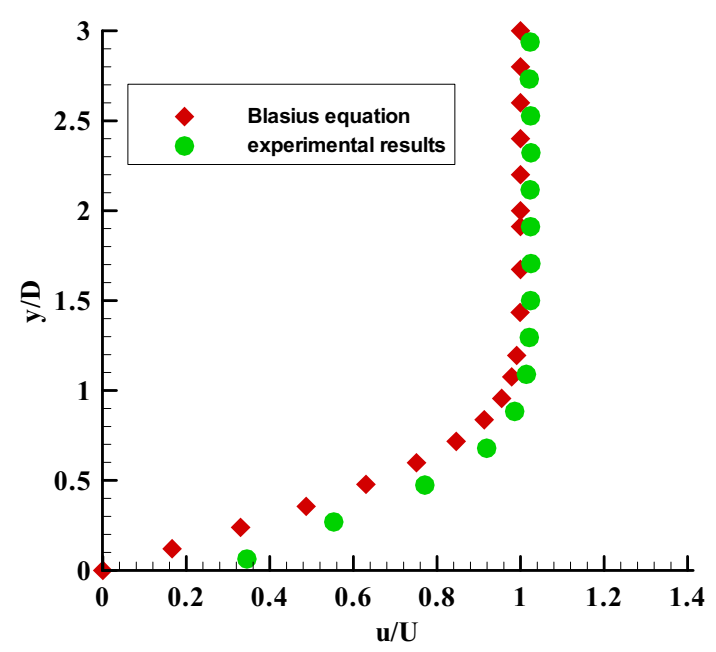

Figure 3. Present experimental data of a flat plate and the Blasius velocity profile at $\operatorname{Re}=32,000$. (outliers) in the image, which was modified by the local conditioning process with $3 \times 3$ kernel size. Seeding particle size and density are small enough to achieve a sharp peak in cross-correlation. The time lag of laser pulses is set according to the Reynolds number of the main flow, and proper arrangement is made to get good illumination at the projected plane.

\section{Results and discussion}

A trapezoidal rib with three different chamfer angles, namely $15^{\circ}, 18^{\circ}$ and $21^{\circ}$, is placed horizontally on the bottom surface of the tunnel across the test section. The effects of varying chamfer angles are investigated on the flow field by PIV and HWA techniques. The effectiveness of the SJ on the flow field is also explained in terms of timeaveraged and instantaneous velocity, vorticity, coherent flow structure and velocity fluctuations. Detailed measurement on the optimal SJ location is done for chamfer angle $21^{\circ}$. All the measurements are carried out at the rib centreline along the $x-y$ plane. From literature, it is established that the optimum angle of the trapezoidal rib is around $21^{\circ}$ for heat transfer application [15]. However, depending upon the SJ strength and its position, the optimum angle may change. To keep the study precise, the effect of SJ positions is limited for angle $21^{\circ}$.

Figure 4(a) and (b) shows the effect of frequency and amplitude on the maximum velocity of the SJ. The optimum frequency at which the maximum SJ velocity is achieved is $80 \mathrm{~Hz}$ (figure 4a), keeping the amplitude fixed at $4 \mathrm{~V}_{\mathrm{pp}}$ for minimum disturbance of the main flow. It is observed that the centreline velocity of the SJ increases monotonously with an increase in operating amplitude, and it is a strong function of operating frequency. To make the 

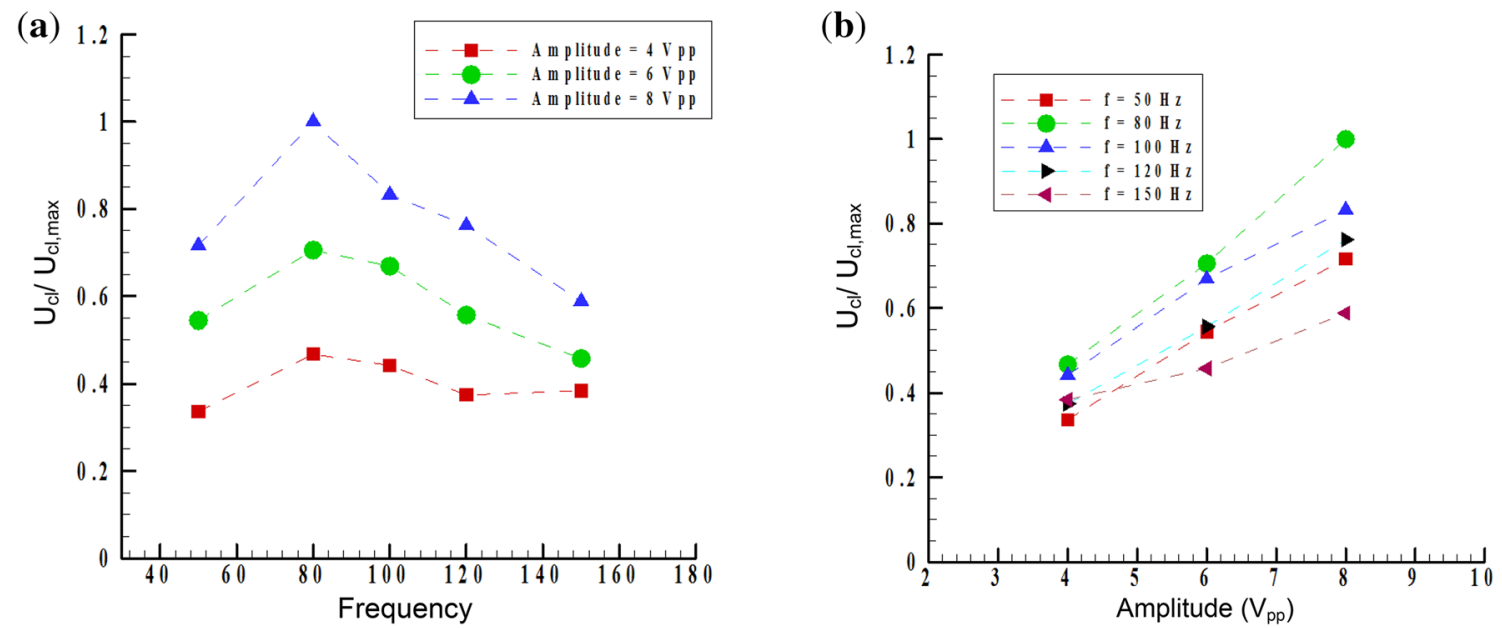

Figure 4. (a) Effect of operating frequency on centreline velocity of synthetic jet at fixed amplitudes. (b) Effect of amplitude of the synthetic jet on centreline velocity at fixed frequencies.

study concise, the SJ location is optimized on the shear layer zone based on maximum influence on the flow field.

\subsection{Time-averaged velocity}

Figure 5 shows the time-averaged velocity contours along with superimposed streamlines for the three chamfer angles $\left(\alpha=15^{\circ}, 18^{\circ}\right.$ and $21^{\circ}$ ). The velocities are normalized with respect to free stream velocity. The top row shows the unmodified rib flow, and the bottom row shows the effect of SJ placed at a fixed location for three different rib geometries. It is observed that the recirculation pattern is a strong function of the chamfer angle. The streamlines show a clear secondary recirculation in the near vicinity of the rib of opposite rotation. The shape and size of this secondary zone are dependent on the chamfer angle. These secondary bubbles faded away with the SJ, and thus helped in eliminating hot spot of the solid rib. It also widens the shear layer, causes the velocity gradient to reduce and increases the velocity magnitude at the recirculation zone. However, as described earlier, reattachment length is not affected much for $21^{\circ}$ chamfer angle as compared with $15^{\circ}$ and $18^{\circ}$. The reattachment length is measured from the leeward corner of the rib to the reattachment point, i.e., the centre of zero-velocity point near the surface. There is a strong agreement between enhancement in Nusselt number and recirculation length [15]. However, the recirculation length was over-predicted from their heat transfer measurement (LCT technique) as compared with PIV measurement.
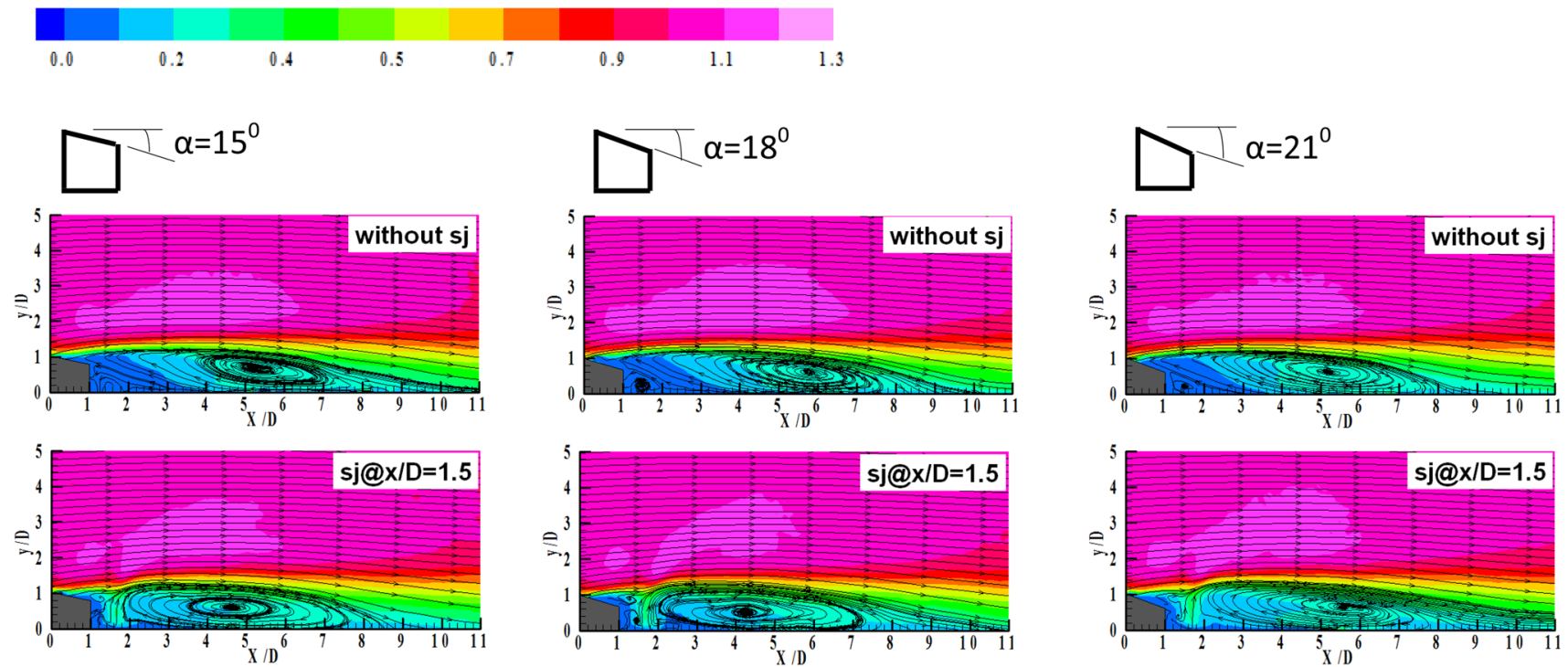

Figure 5. Time-averaged normalized velocity contours with streamlines for different chamfer angles of the rib $\left(15^{\circ}, 18^{\circ}\right.$ and $\left.21^{\circ}\right)$ with synthetic jet located at fixed downstream position $x / D=1.5$. 
Overall, there was around $20 \%$ enhancement in heat transfer with the rib. Maximum heat transfer was seen immediately downstream of the reattachment point. The reason is the sweeping of the shear layer, which carries the heat out from the surface. With the SJ, the sweeping action starts early as the reattachment point moves closer to the rib. The secondary bubble plays a significant role in heat transfer from the leeward corner of the rib.

The eddies generated from the synthetic jet interrupt the shear layer in the separated region, which leads to change in the reattachment length.

The reattachment length is a significant quantity as far as the heat transfer enhancement and the pressure penalty are of concern. With the decrease in recirculation length, the adverse pressure gradient zone reduces, which means less pumping power is required for the same heat transfer enhancement. The recirculation bubble shifts towards the leeward corner for $15^{\circ}$ and $18^{\circ}$ chamfer angles and hence reduces the reattachment length, while $21^{\circ}$ chamfer angles do not show any significant effect of SJ for this location.

Figure 6 shows the effect of SJ position on the flow behaviour for $21^{\circ}$ chamfer angle. With the SJ located upstream of the rib $(x / D=-1.5)$, it creates a dual centre of the recirculation bubble and tends to diminish as it approaches rib's front face. However, for the downstream locations of the SJ, no such dual-centre recirculation bubbles are seen. The adjacent rib flow structure is modified substantially, which is observed from near-wake streamline patterns. Figure 7 shows the time-averaged transverse velocity ( $v$-velocity) profiles at three different $x$-locations.

There is a significant change in $v$-velocity component away from the rib, and also it is a strong function of the SJ position. The maximum magnitude is observed at a certain distance from the wall and the rib surface. There is a strong relationship between $v$-velocity and heat transfers as the mixing gets enhanced with transverse velocity. Table 1 shows the reattachment length for a different combination of rib geometry and SJ location with respect to the rib. Maximum reduction in the reattachment length is observed for $21^{\circ}$ chamfer angle rib with the SJ position at $x / D=-0.5$.

\subsection{Vorticity distribution}

Introducing an SJ either upstream or downstream of the trapezoidal rib influences the vorticity structure behind the rib (figure 8). The vortices generated from the SJ interact with the shear layer of the rib; as a result, the wake zone behind the rib is modified. The dark blue colour contours show the maximum magnitude of vorticity. Without the SJ, it extends up to $7 D$ downstream from the rib centre. With the SJ, it extends up to 5-6D. This ultimately reduces the pressure penalty by reducing the wake zone.

Figure 8 shows the time-averaged normalized vorticity distribution behind three different chamfer angle ribs and the effect of SJ. Prior to incorporating the SJ in the flow,

\begin{tabular}{|llllllll|}
\hline 0.0 & 0.2 & 0.4 & 0.5 & 0.7 & 0.9 & 1.1 & 1.3
\end{tabular}
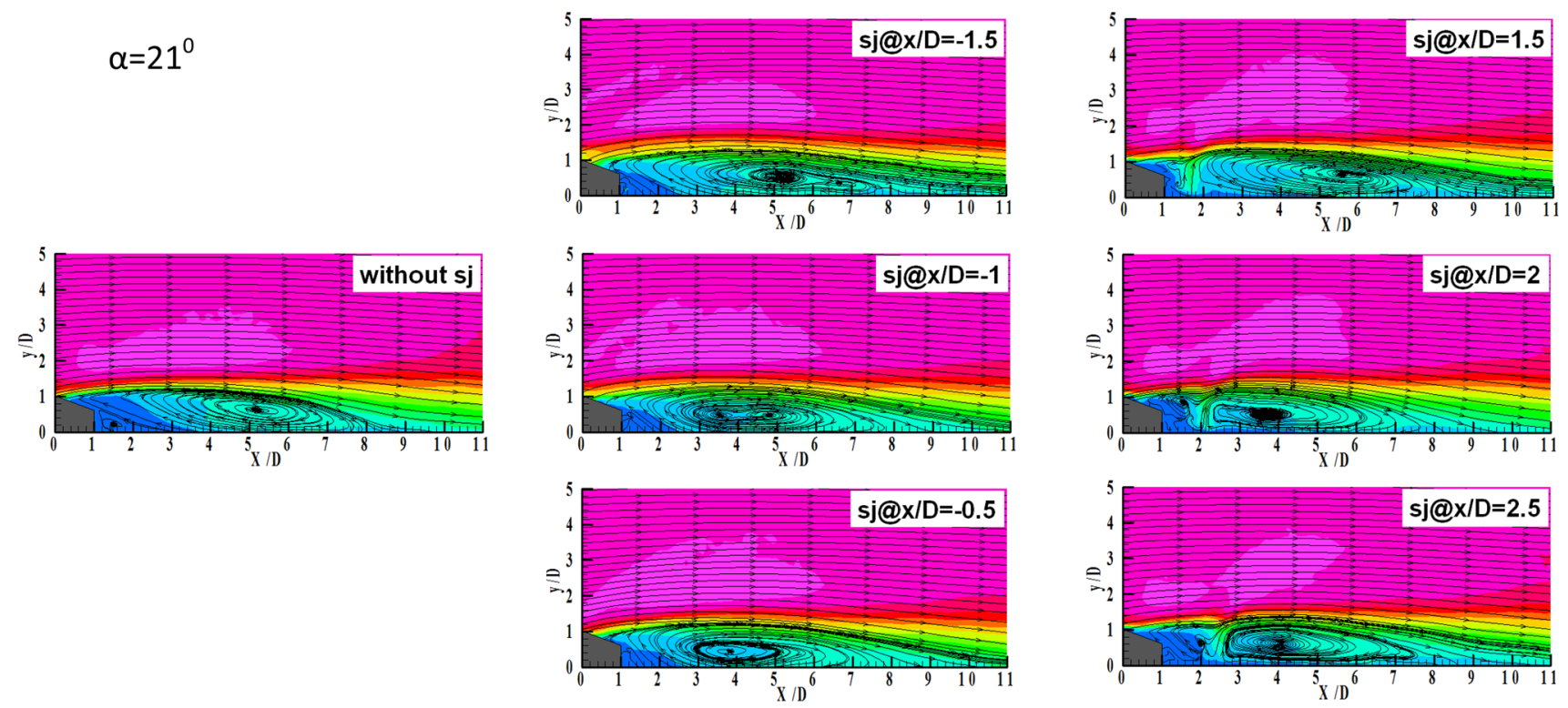

Figure 6. Time-averaged normalized velocity contours with superimposed streamlines for a rib $\left(\alpha=21^{\circ}\right)$ with different locations of synthetic jet upstream and downstream of the rib (operating frequency and amplitude of synthetic jet fixed at $80 \mathrm{~Hz}$ and $4 \mathrm{~V}_{\mathrm{pp}}$, respectively). 

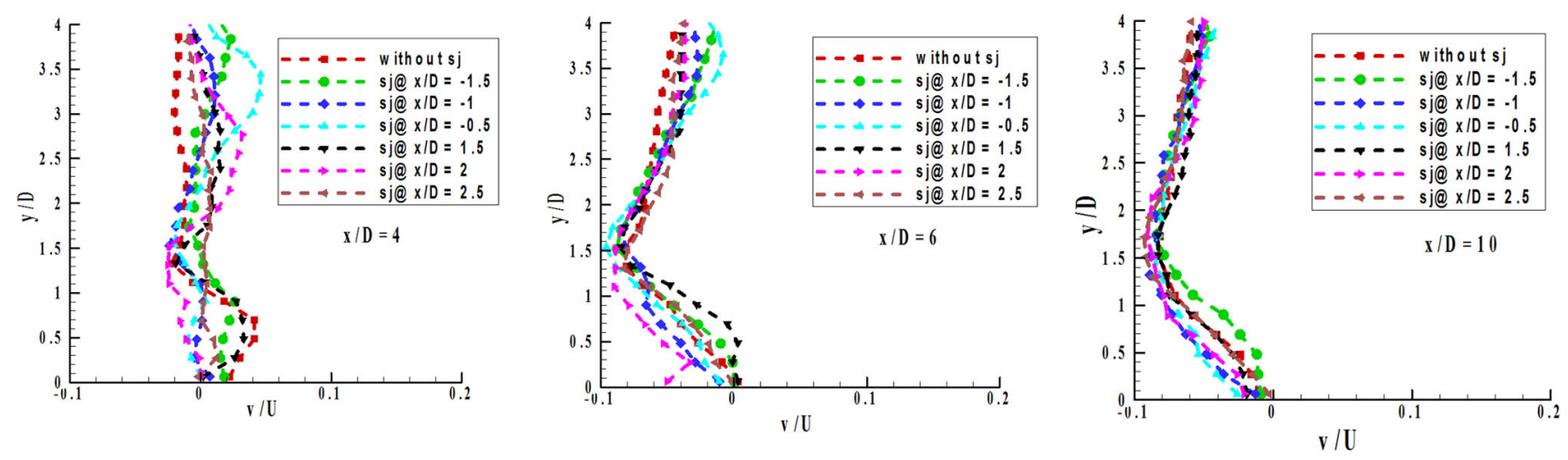

Figure 7. Time-averaged $v$-velocity profiles on three different planes behind the rib (chamfer angle $\alpha=21^{\circ}$ ) for various synthetic jet locations.

Table 1. Reattachment length for all different ribs and the effect of synthetic jet.

\begin{tabular}{|c|c|c|c|}
\hline Sl. no. & Rib shape & Synthetic jet location & Reattachment length, cm \\
\hline \multirow[t]{2}{*}{1.} & Trapezoidal with chamfer angle $15^{\circ}$ & Without synthetic jet & 5.3 \\
\hline & & Synthetic jet @x/D=1.5 & 4.7 \\
\hline \multirow[t]{2}{*}{2.} & Trapezoidal with chamfer angle $18^{\circ}$ & Without synthetic jet & 5.8 \\
\hline & & Synthetic jet @x/D=1.5 & 4.3 \\
\hline \multirow[t]{7}{*}{3.} & Trapezoidal with chamfer angle $21^{\circ}$ & Without synthetic jet & 5.2 \\
\hline & & Synthetic jet @ $x / D=-1.5$ & 3.5 \\
\hline & & Synthetic jet @ $x / D=-1$ & 3.8 and 4.8 \\
\hline & & Synthetic jet @ $@ / D=-0.5$ & 3.8 \\
\hline & & Synthetic jet @ $x / D=1.5$ & 5.5 \\
\hline & & Synthetic jet @ $x / D=2$ & 3.8 \\
\hline & & Synthetic jet @x/D=2.5 & 4.0 \\
\hline
\end{tabular}

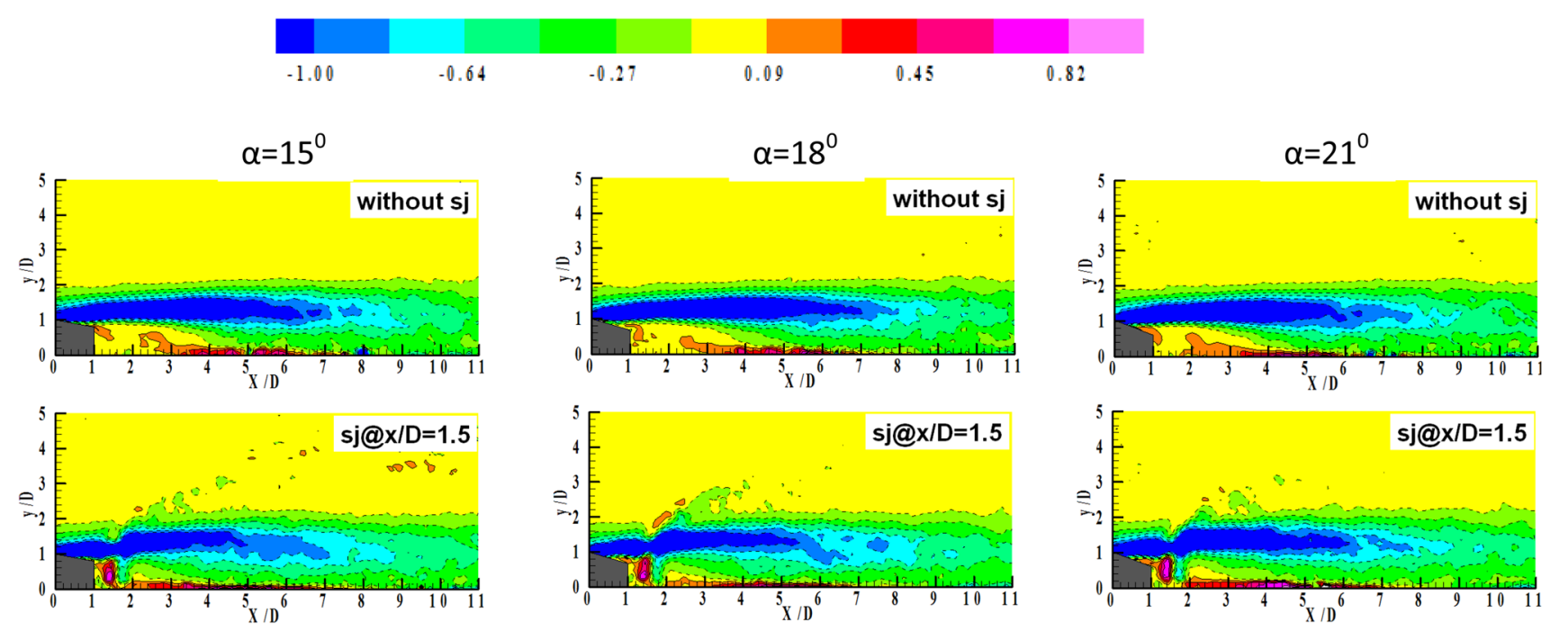

Figure 8. Time-averaged non-dimensionalized span-wise vorticity contours $\left(\omega_{z} D / U\right)$ for different chamfer angles of trapezoidal rib $\left(15^{\circ}, 18^{\circ}\right.$ and $\left.21^{\circ}\right)$ with the synthetic jet located at fixed downstream position $x / D=1.5$.

negative sign vortices (counter-clockwise) are generated as the separation of flow occurs with shear layer formation. It widens in the flow direction up to $10 D$ as the strong vorticity in the near wake diffuses downstream. With the SJ the diffusion process starts early, as the primary vortices interact with the vortices generated from the SJ. 
This is due to the interaction of SJ vortices (both clockwise and counter-clockwise) with the vortices generated in the separated shear layer dominated by clockwise vortices (negative sign). Figure 9 shows the time-averaged contours behind the $21^{\circ}$ trapezoidal ribs and the effect of the SJ. With the SJ the unsteadiness, as well as strength in the large scale vorticity structure, increases substantially in the near wall and far wake region from the wall. Further, when the SJ is placed upstream of the rib (figure 10), the shear layer becomes highly unstable, and the diffusion is stronger in the near wake. With the SJ, the breakdown of the large scale structure and better mixing in the adjacent wake zone of the rib started.

Moreover, these vortices deflected in the upward direction and as a result, the diffusion process became stronger in the near wake. However, the vorticity distribution is least sensitive to SJ location. Due to this high concentration of vortices, the diffusion mechanism enhances the heat transfer from the wall region.

\subsection{Turbulence intensity, turbulent stresses}

Figure 11 displays the distribution of turbulence intensity in the near wake of the trapezoidal rib for three different chamfer angles. Top images show the effect of chamfer angle, while bottom images show the corresponding impact of the SJ. With the SJ, the fluctuating flow field alters specifically in the near-wake zone.
With the SJ, the maximum intensity shifted close to the rib and spread over a larger near-wake zone. It is beneficial as it increases the heat transfer rate from the surface. In addition to this, a noticeable variation in the distribution of turbulence intensity is observed when the SJ is placed upstream of the rib. The turbulence spreads over a broader wake region, and hence more mixing occurs (figure 12).

However, the most noticeable effect of SJ on turbulence intensity is at its downstream location due to enhanced diffusion of the shear layers and its turbulence count at the near zone of the orifice. To be specific, the SJ at $2 D$ downstream from the front face of the rib shows maximum turbulence as compared with the other two locations downstream studied. The distributions of $u_{\mathrm{rms}}$ and $v_{\mathrm{rms}}$ are shown in figures 13 and 14, respectively. They represent the normal stresses in $x$ - and $y$-direction. Both components $\left(u_{\mathrm{rms}}\right.$ and $\left.v_{\mathrm{rms}}\right)$ increase with the distance from the wall, reach a maximum value in the shear layer core and then reduce to free stream velocity. The velocity magnitude of $u_{\mathrm{rms}}$ is higher than the $v_{\mathrm{rms}}$ value for all the locations studied. The stream-wise normal stress is related to the transverse velocity gradient in the flow direction, whereas $v_{\text {rms }}$ is related to the pressure gradient between the wake zone and outside the free stream. The maximum normal stress in the flow direction $\left(u_{\mathrm{rms}}\right)$ is observed at $x / d=6.0$. However, the effect of the SJ is visible only after a certain distance normal to the wall.

At a higher stream-wise distance, the velocity takes a self-similar form and the effect of the SJ is negligible. In this sense, the effect of the SJ is local. The impact of the SJ
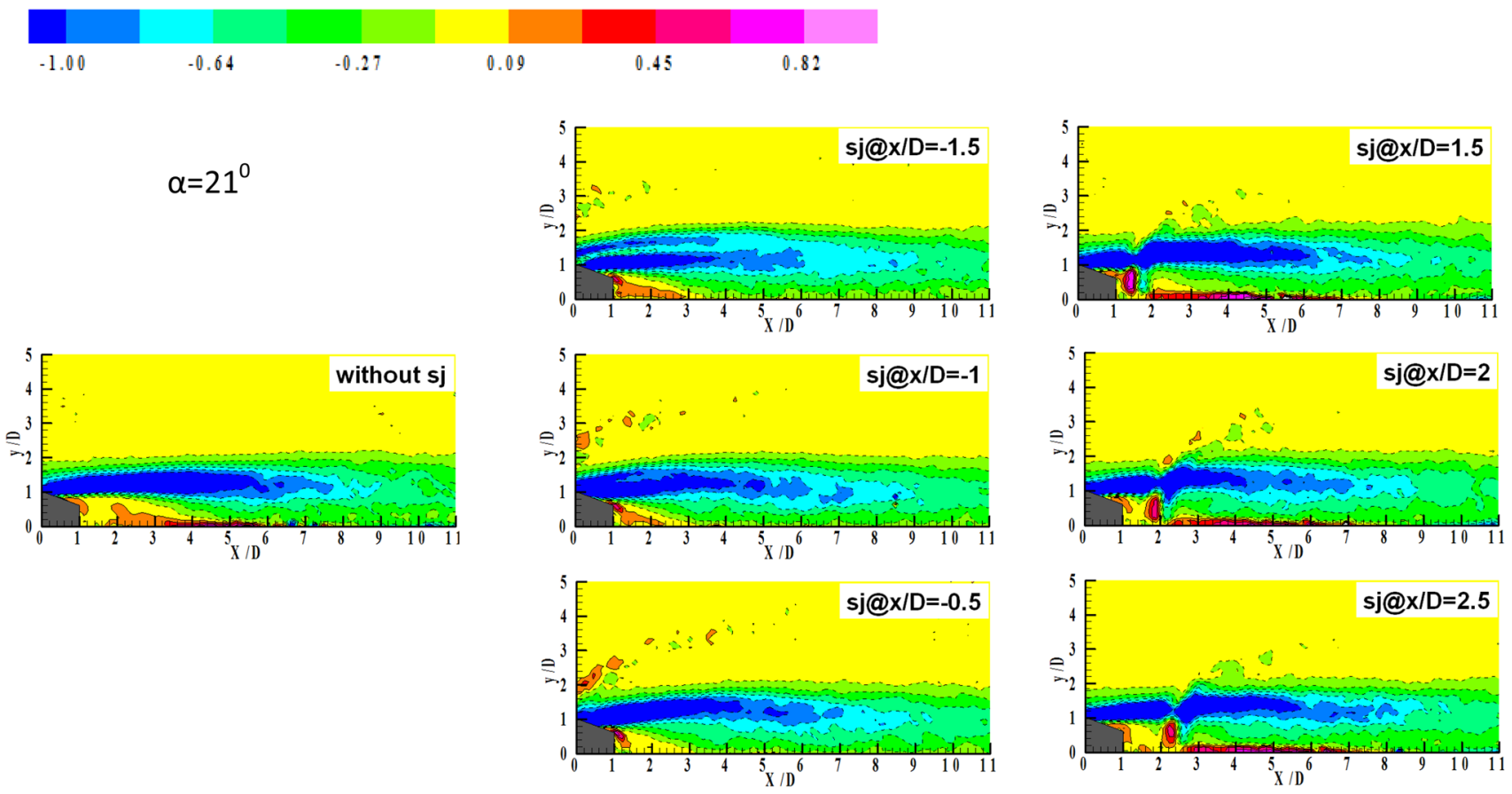

Figure 9. Time-averaged non-dimensionalized span-wise vorticity contours $\left(\omega_{z} D / U\right)$ for a trapezoidal rib $\left(\alpha=21^{\circ}\right)$ with different locations of synthetic jet upstream and downstream of the rib. 

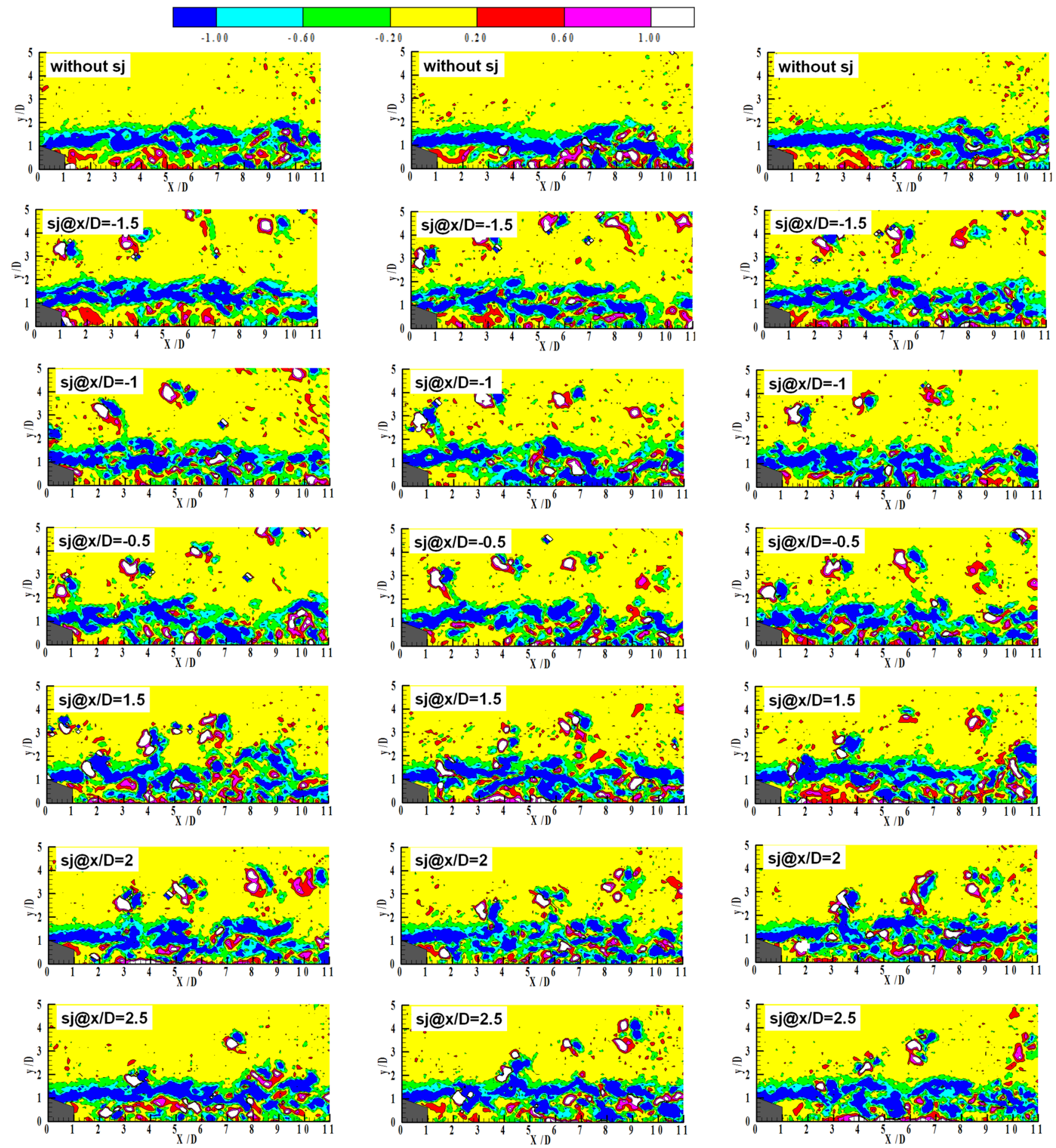

Figure 10. Instantaneous vorticity contours for different locations of synthetic jet for trapezoidal $21^{\circ}$ rib. The top row shows the vorticity structure behind unmodified rib arrangement.

downstream is more useful to increase the heat transfer from the surface due to its high intensity near the wall, while the upstream location of SJ is more beneficial for reduction of pressure penalty as it energizes the flow approaching the rib geometry.

The $v_{\text {rms }}$ component can be correlated with the pressure gradient within and outside the wake zone. With the SJ, the $v_{\mathrm{rms}}$ spreads over a wider zone and normalizes the pressure gradient.

\subsection{Reynolds stress}

Figures 15 and 16 show the normalized Reynolds stress behind a trapezoidal rib at different chamfer angles and the 

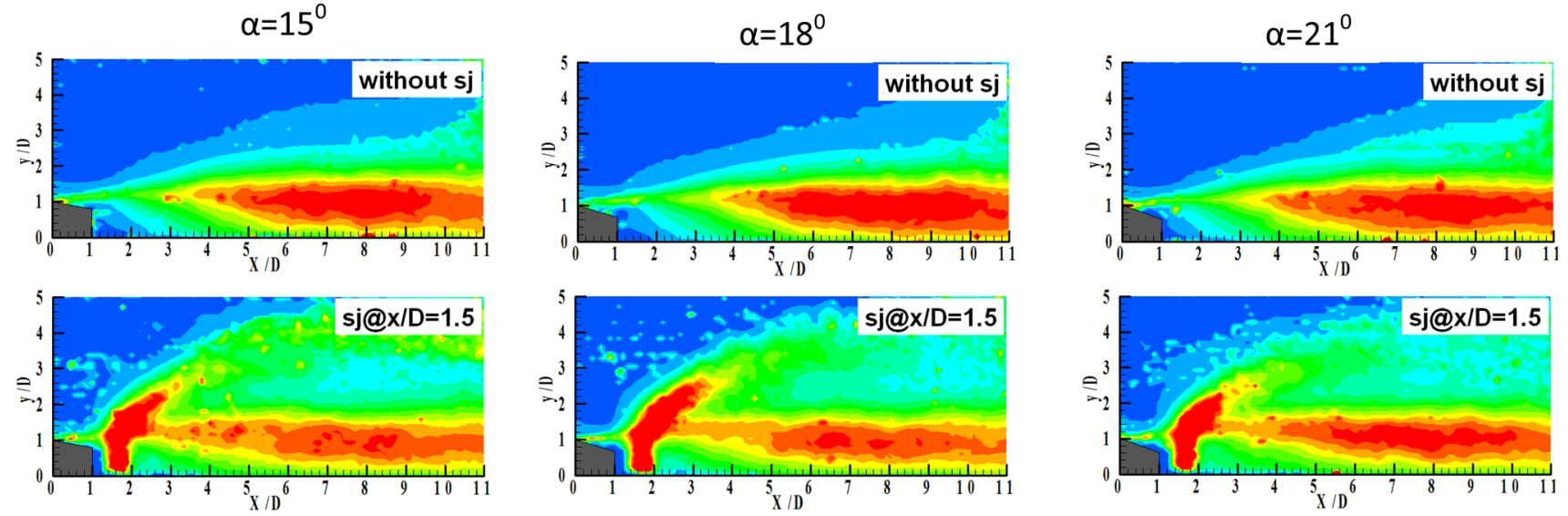

Figure 11. Time-averaged non-dimensionalized turbulence intensity $\left(u_{\mathrm{rms}}^{2}+v_{\mathrm{rms}}^{2}\right)^{0.5} / U$ for different chamfer angle trapezoidal ribs $\left(15^{\circ}, 18^{\circ}, 21^{\circ}\right)$ with the synthetic jet located at fixed downstream position $x / D=1.5$.

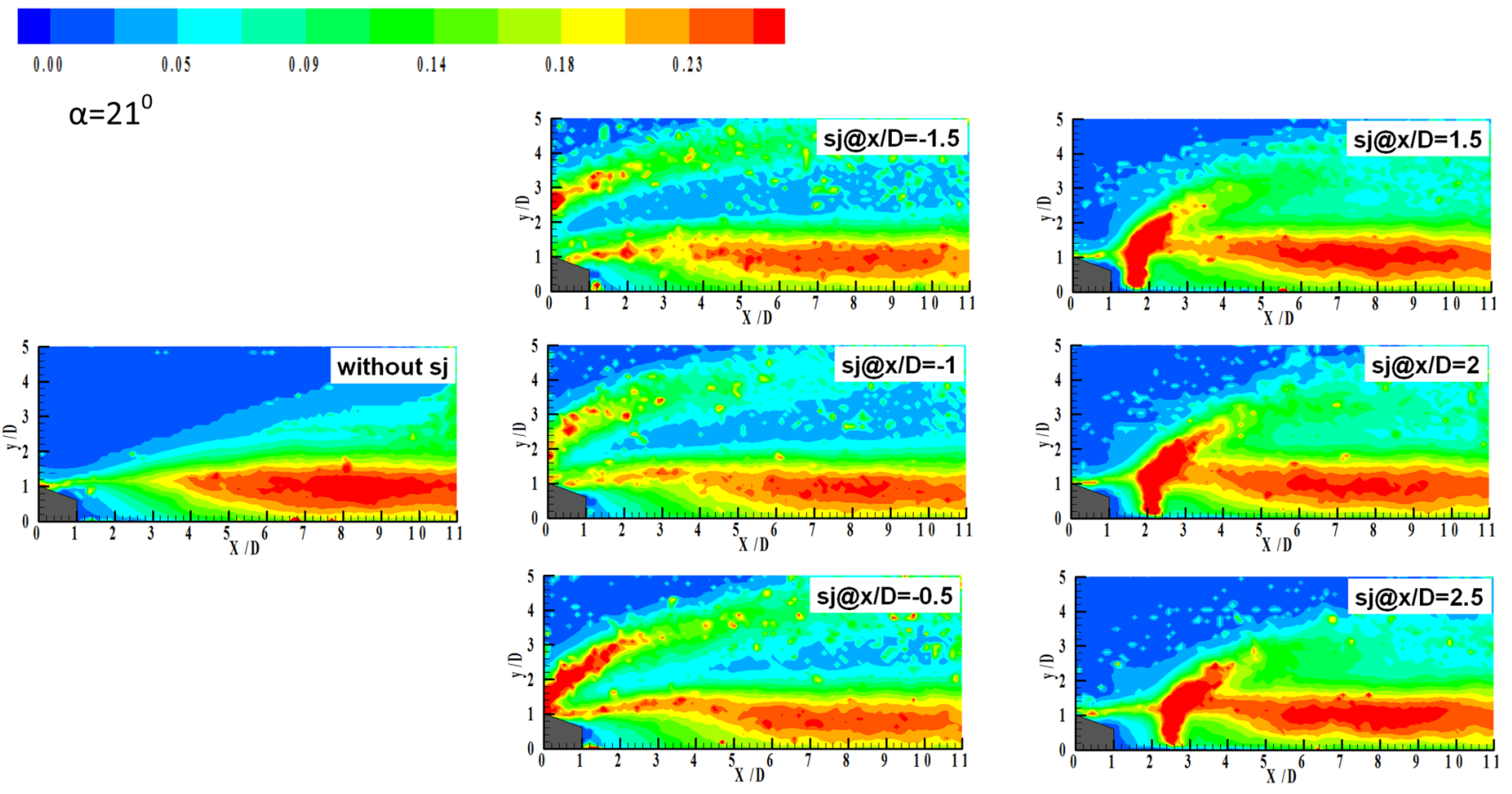

Figure 12. Time-averaged non-dimensionalized turbulence intensity $\left(u_{\mathrm{rms}}^{2}+v_{\mathrm{rms}}^{2}\right)^{0.5} / U$ for a trapezoidal rib $\left(\alpha=21^{\circ}\right)$ with different locations of synthetic jet upstream and downstream of the rib.

effect of SJ. Similar to velocity fluctuations ( $u_{\mathrm{rms}}$ and $\left.v_{\mathrm{rms}}\right)$, the Reynolds stress also has a maximum magnitude in the shear layer and reduces to a minimum value outside the shear layers. The production of the shear stress is related to the velocity gradient in the flow field. This shear stress increases the wall friction and heat transfer from the wall. With the position of the SJ, the maximum shear stress value shifted downwards.

\subsection{Power spectra}

Figure 17 shows the power spectra of the stream-wise velocity signal for trapezoidal $21^{\circ}$ ribs with the effect of SJ location. Spectra are calculated from a long time signal (20 s) at a sampling rate of $1000 \mathrm{~Hz}$. The minimum and maximum frequencies resolved are, respectively, 0.05 and $500 \mathrm{~Hz}$. This is sufficient to capture a wide range of 

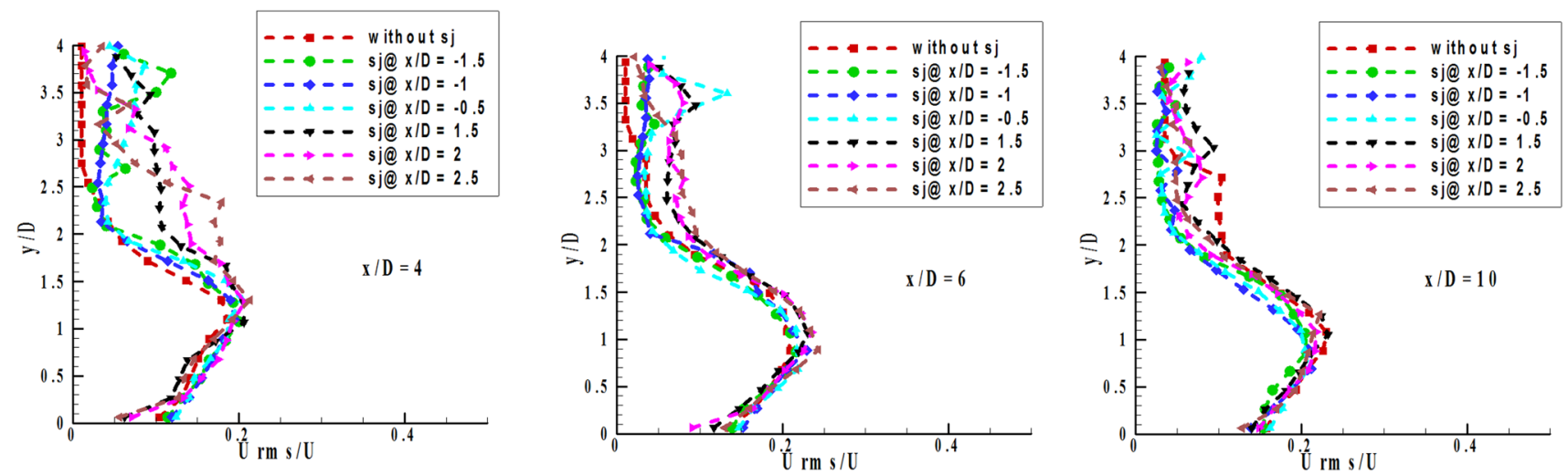

Figure 13. Non-dimensionalized $u_{\mathrm{rms}}$ profile in vertical direction on three different planes behind the rib (trapezoidal rib with chamfer angle $\alpha=21^{\circ}$ ) for various synthetic jet locations.
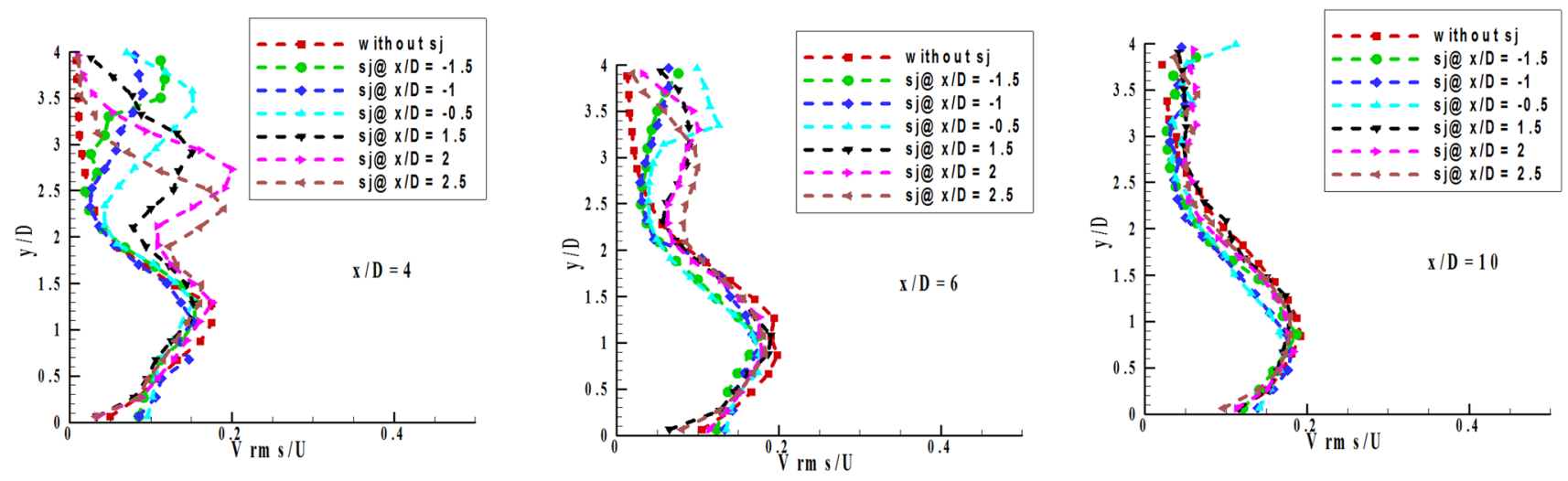

Figure 14. Non-dimensionalized $v_{\text {rms }}$ profile in vertical direction on three different planes behind the trapezoidal rib with chamfer angle $\alpha=21^{\circ}$ for various synthetic jet locations.

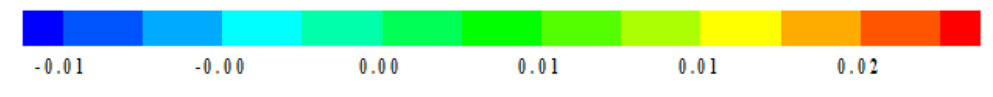

$$
\alpha=15^{0}
$$
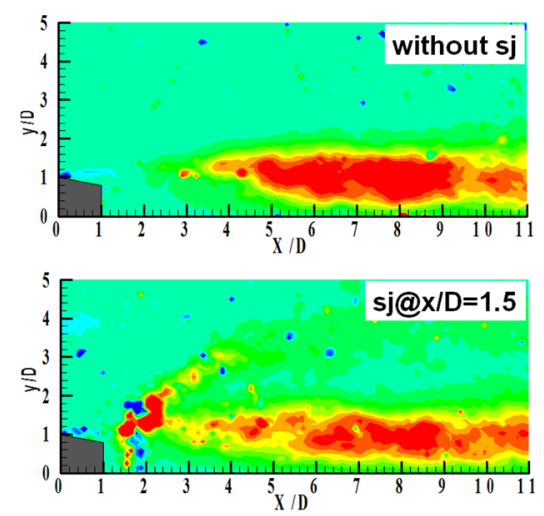
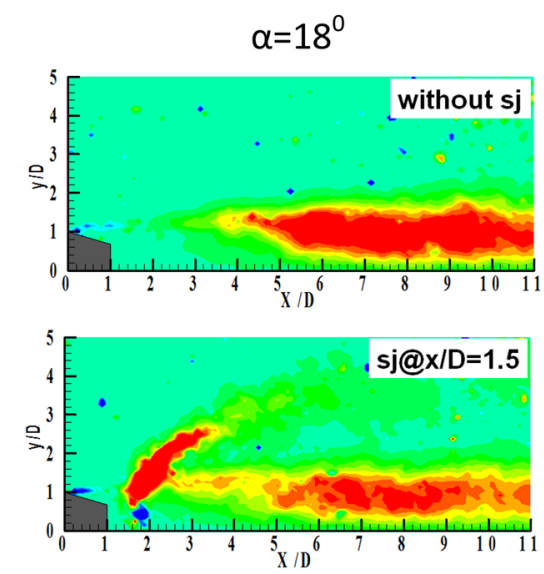
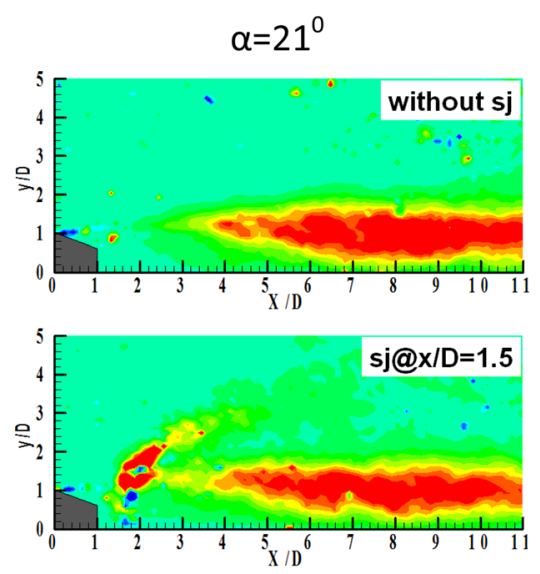

Figure 15. Time-averaged non-dimensionalized Reynolds stress $\left(-u^{1} v^{1} / U^{2}\right)$ for different chamfer angle trapezoidal ribs $\left(15^{\circ}, 18^{\circ}, 21^{\circ}\right)$ with synthetic jet located at fixed downstream position $x / D=1.5$. 

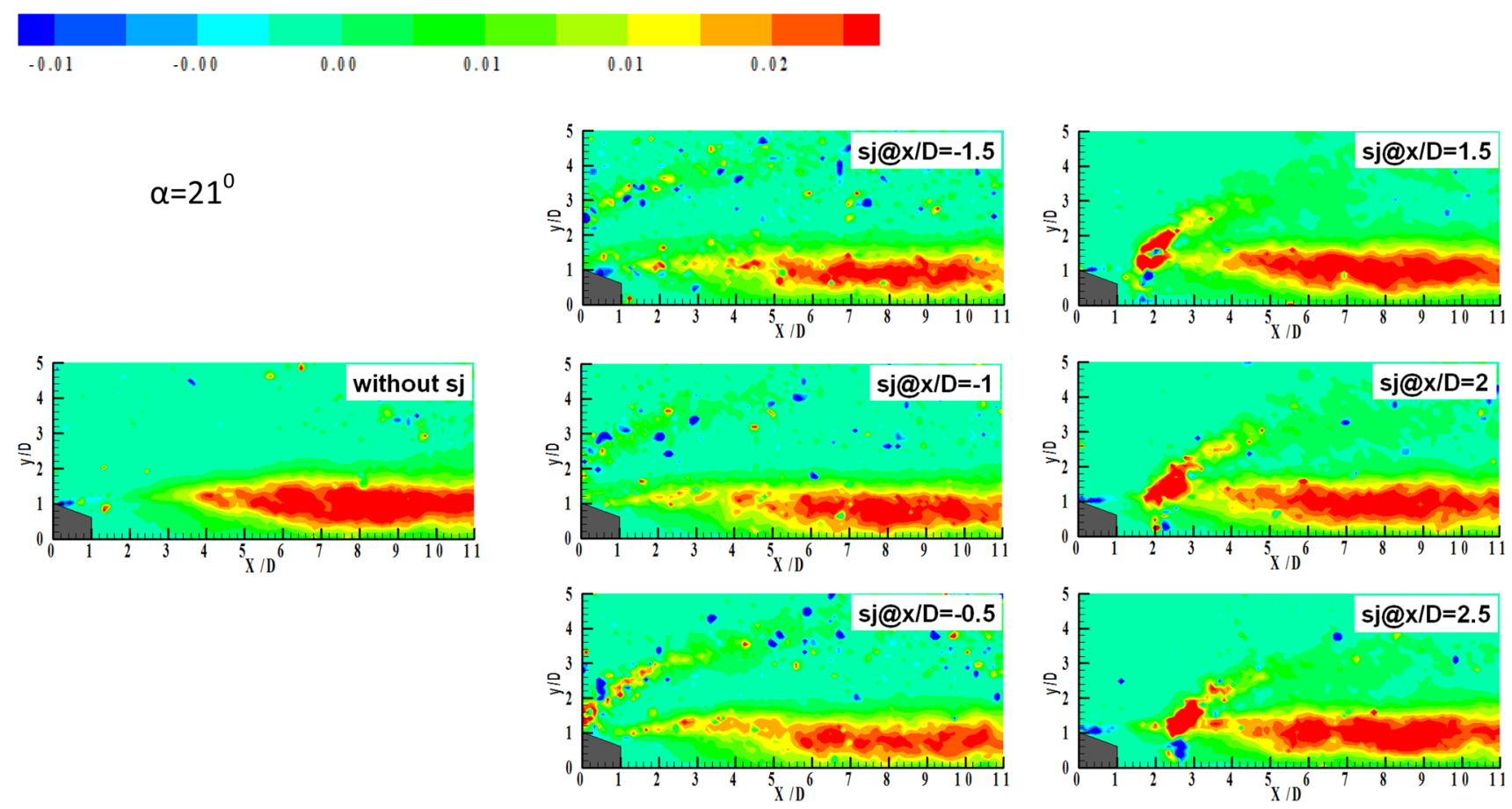

Figure 16. Time-averaged non-dimensionalized Reynolds stress $\left(-u^{1} v^{1} / U^{2}\right)$ for a trapezoidal rib $\left(\alpha=21^{\circ}\right)$ with synthetic jet located at different locations upstream and downstream of the rib.
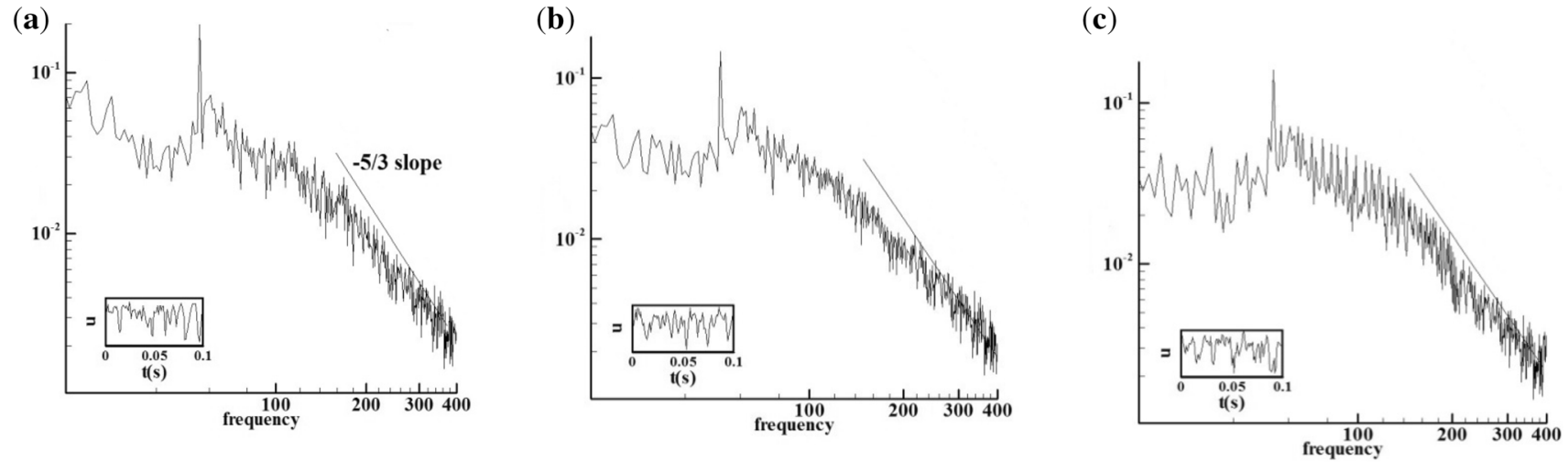

Figure 17. Power spectrum for trapezoidal rib with chamfer angle $21^{\circ}$ (a) without synthetic jet, (b) with synthetic jet located $1 D$ upstream from the front face of rib and (c) with synthetic jet located $1.5 D$ downstream from the front face of rib.

frequencies present in the signal. The voltage signal is converted to a velocity signal using calibration.

The spectra have been normalized with the area under the curve, which equals the mean square velocity fluctuations of the flow. The spectra give the distribution of large scale and small scale eddies and their kinetic energy. The periodic fluctuation in the separated shear layer region is shown as the peak in the spectra. The spectra in the subinertial ranges having a negative slope close to $-5 / 3$ shows that the flow is highly three dimensional. This results in higher mixing and maximum heat transfer. The flow periodicity is visible from the time signal $(0.1 \mathrm{~s})$ superimposed in the spectra.

\section{Conclusion}

The present study investigates the flow behaviour behind trapezoidal ribs for three different chamfer angles $\left(15^{\circ}, 18^{\circ}\right.$ and $21^{\circ}$ ) and the effect of the SJ, which is flush-mounted on the bottom surface of the test section. The major difference in the flow structure without introducing the SJ is observed in terms of recirculation length and secondary bubble formation for these three chamfer angles. However, a detailed study has been done at $21^{\circ}$ angle from a heat transfer perspective, which has been optimized by various authors. The impact of locations of the SJ with respect to rib location is studied for chamfer angle $21^{\circ}$. The Reynolds number 
is kept fixed at 32,000 based on hydraulic diameter. PIV and HWA are used to characterize the flow field in terms of mean and fluctuating velocities. The main focus is on nearwake zone of the rib. The recirculation length has substantially reduced with the SJ. The secondary flow, which is evident behind the ribs, is eliminated with the SJ and thus it avoids the probable hot spot in the vicinity of the rib. A remarkable change in flow phenomena such as recirculation length and turbulence intensity is observed when the location of the SJ is optimized. The effect of the chamfer angle on the recirculation length is not prominent. However, with the SJ, maximum $27 \%$ reduction in recirculation length is observed in the wake of the ribs. The region of the low-velocity zone is reduced with the SJ, which will be helpful in heat transfer enhancement and reduces pressure penalty.

\section{Nomenclature}

A operating amplitude of synthetic jet, $\mathrm{m}^{2}$

$d \quad$ orifice diameter of synthetic jet, $\mathrm{m}$

$D$ bottom edge dimension of the rib, $\mathrm{m}$

$f \quad$ operating frequency of synthetic jet, $\mathrm{Hz}$

$f_{0} \quad$ resonance frequency/optimum frequency/ frequency for maximum average velocity, $\mathrm{Hz}$

$H \quad$ height of tunnel, m

$\alpha \quad$ chamfer angle of trapezoidal rib

Re Reynolds number $\left(\rho U D_{\mathrm{h}} / \mu\right)$

$D_{\mathrm{h}} \quad$ hydraulic diameter of tunnel, $\mathrm{m}$

SJ synthetic jet

$U_{\mathrm{cl}} \quad$ time-average centreline velocity of synthetic jet, $\mathrm{m} / \mathrm{s}$

$U_{\mathrm{cl}, \max }$ time-average centreline velocity of synthetic jet at its maximum (for $80-\mathrm{Hz}$ frequency)

$U$ free stream velocity, $\mathrm{m} / \mathrm{s}$

$u \quad$ local stream-wise velocity, $\mathrm{m} / \mathrm{s}$

$x \quad$ distance in stream-wise direction, $\mathrm{m}$

$y \quad$ distance in transverse of stream direction, $m$

$u_{\mathrm{rms}} \quad$ root mean square velocity fluctuation in flow direction, $\mathrm{m} / \mathrm{s}$

$v_{\text {rms }} \quad$ root mean square velocity fluctuation in transverse of flow direction, $\mathrm{m} / \mathrm{s}$

\section{References}

[1] Glezer A, Allen M G, Coe D J, Smith B L, Trautman M A and Wiltse J W 1998 U.S. Patent No. 5,758,823. Washington, DC: U.S. Patent and Trademark Office
[2] Chaudhari M, Puranik B and Agrawal A 2010 Heat transfer characteristics of synthetic jet impingement cooling. Int. J. Heat Mass Transf. 53(5): 1057-1069

[3] Mcguinn A and Persoons T 2013 Flow regime characterization of an impinging axisymmetric synthetic jet. Exp. Therm. Fluid Sci. 47: 241-251

[4] Nani D J and Smith B L 2012 Effect of orifice inner lip radius on synthetic jet efficiency. Phys. Fluids 24(11): 115110

[5] Wang J, Shan R, Zhang C and Feng L 2010 Experimental investigation of a novel two-dimensional synthetic jet. Eur. J. Mech. B: Fluids 29(5): 342-350

[6] Chaudhari M, Verma G, Puranik B and Agrawal A 2009 Frequency response of synthetic jet cavity. Exp. Therm. Fluid Sci. 33: 439-448

[7] Jain M, Puranik B and Agrawal A 2011 A numerical investigation of effects of cavity and orifice parameters on the characteristics of a synthetic jet flow. Sens. Actuat. A 165: 351-366

[8] Krishnan G and Mohseni K 2009 An experimental and analytical investigation of rectangular synthetic jets. J. Fluid Eng. 131(12): 121101

[9] Tang H and Zhong S 2007 A static compressible flow model of synthetic jet actuators. Aeronaut. J. 111(1121): 421-431

[10] Feng L H and Wang J J 2014 Modification of a circular cylinder wake with synthetic jet: vortex shedding modes and mechanism. Eur. J. Mech. B: Fluids 43: 14-32

[11] Xia Q and Zhong S 2014 Enhancement of laminar flow mixing using a pair of staggered lateral synthetic jets. Sens. Actuat. A: Phys. 207: 75-83

[12] Tariq A, Panigrahi P K and Muralidhar K 2004 Flow and heat transfer in the wake of a surface-mounted rib with a slit. Exp. Fluids 37(5): 701-719

[13] Panigrahi P K and Acharya S 2004 The flow over a surface mounted rib turbulator under single-mode and dual-mode excitation. J. Wind Eng. Ind. Aerodyn. 92(14): 1219-1244

[14] Panigrahi P K and Acharya S 2005 Excited turbulent flow behind a square rib. J. Fluids Struct. 20(2): 235-253

[15] Ali M S, Tariq A and Gandhi B K 2013 Flow and heat transfer investigation behind trapezoidal rib using PIV and LCT measurements. Exp. Fluids 54: 1520

[16] Qayoum A, Gupta V, Panigrahi P K and Muralidhar K 2010 Perturbation of a laminar boundary layer by a synthetic jet for heat transfer enhancement. Int J. Heat Mass Transf. 53(23): 5035-5057

[17] Chauhan M K, Dutta S, Gandhi B K and More B S 2016 Experimental investigation of flow over a transversely oscillating square cylinder at intermediate Reynolds number. J. Fluid Eng. 138(5): 051105

[18] Dutta S, Panigrahi P K and Muralidhar K 2008 Experimental investigation of flow past a square cylinder at an angle of incidence. J. Eng. Mech. 134(9): 788-803

[19] More B S, Dutta S, Chauhan M K and Gandhi B K 2015 Experimental investigation of flow field behind two tandem square cylinders with oscillating upstream cylinder. Exp. Therm. Fluid Sci. 68: 339-358 